\title{
Optimized 3D synthetic aperture for controlled-source electromagnetics
}

\author{
Allison Knaak ${ }^{1}$, Roel Snieder ${ }^{2}$, Liam Ó. Súilleabháin ${ }^{3}$, \\ Yuanzhong $\mathrm{Fan}^{3}$, and David Ramirez-Mejia ${ }^{3}$
}

\begin{abstract}
Locating offshore hydrocarbon reservoirs has become more challenging with smaller, deeper, or shallow-water targets in complicated environments. Controlled-source electromagnetics (CSEM) is a geophysical method used to find reservoirs in marine settings. The diffusive nature of CSEM fields means that the signal from the target is only a small part of the total field. To reduce the impact of the complicated settings and to improve the detecting capabilities of CSEM, we have applied a synthetic aperture to CSEM data. The synthetic aperture virtually increased the length and width of the CSEM source by combining the responses from multiple individual sources. Applying a weight to each source steered or focused the synthetic aperture source array in the inline and crossline directions. We have developed an optimization method to find the optimal weights for synthetic aperture arrays that adapted to the information about the reservoirs in the CSEM data. To demonstrate the benefits of a weighted synthetic aperture, we have applied a 2D synthetic aperture array and a crossline-only synthetic aperture array to noisy, simulated electromagnetic fields. Both synthetic aperture arrays reduced the noise and increased the detectability of the modeled reservoirs when compared with the original CSEM response. The crossline-only synthetic aperture array also preserved the structural information within the measurements.
\end{abstract}

\section{INTRODUCTION}

Controlled-source electromagnetics (CSEM), a geophysical method used primarily for finding oil reservoirs in marine settings, was first proposed in academic research and was implemented in industry more than a decade ago (see Eidesmo et al. [2002], Edwards [2005], Constable and Srnka [2007], and Constable [2010] for history and an overview). The method is now used widely to derisk and discover offshore reservoirs (Constable and Srnka, 2007). The method involves towing an electric dipole source over receivers placed on the ocean floor, which record the electric and magnetic fields. The dipole source, operating at low frequencies (typically approximately $0.1-1 \mathrm{~Hz}$ ), emits a signal, which travels down through the conductive subsurface creating diffusive fields (Constable and Srnka, 2007). The diffusive fields decay quickly, which means that the signal from the reservoir is only a small part of the total field. The difficulty of identifying the signal from the reservoir is exacerbated in complicated environments. Finding and derisking reservoirs with CSEM has become more challenging because CSEM is applied to targets that are deeper, smaller, and in more complex settings. We apply a synthetic aperture to reduce the impact of these issues and to improve the detecting capabilities of CSEM.

Researchers in the radar field first developed the synthetic aperture, and now many different fields, including medical imaging and geophysics, apply the technique (Van Veen and Buckley, 1988; Jensen et al., 2006). The synthetic aperture uses information from multiple individual sources to create a source array with a longer aperture. Fan et al. (2010) first apply a synthetic aperture to CSEM fields using sources from a single towline to create a source array several kilometers long. The use of a synthetic aperture has expanded to include sources from multiple towlines, which allows for the creation of a 2D source array composed of multiple sources in the inline and crossline directions. We define the axis parallel to the towing direction as the inline axis and the axis perpendicular to the towing direction as the crossline axis. We give a weight to each source in the synthetic aperture source array to maximize the signal from the reservoir. The weighting is analogous to beamforming with

Manuscript received by the Editor 29 July 2014; revised manuscript received 30 May 2015; published online 23 September 2015.

${ }^{1}$ Colorado School of Mines, Department of Geophysics, Center for Wave Phenomena, Golden, Colorado, USA and Shell International Exploration \& Production, Houston, Texas, USA. E-mail: aknaak@mines.edu.

${ }^{2}$ Colorado School of Mines, Department of Geophysics, Center for Wave Phenomena, Golden, Colorado, USA. E-mail: rsnieder@mines.edu.

${ }^{3}$ Shell International Exploration \& Production, Houston, Texas, USA. E-mail: liam.osuilleabhain@ @hell.com; yuanzhong.fan@shell.com; david. ramirezmejia@shell.com.

(C) 2015 Society of Exploration Geophysicists. All rights reserved. 
synthetic aperture radar and allows us to steer or focus the energy in the inline, crossline, or both directions. In this article, we first review the application of weighted synthetic aperture to CSEM. Then, we introduce a method to find the optimal weighting parameters for a synthetic aperture source array. Finally, we present two examples of applying the optimal weighted synthetic aperture to synthetic electromagnetic fields with noise added.

\section{WEIGHTED SYNTHETIC APERTURE}

We review the theory and history of weighted synthetic aperture and present a new weighting formulation for applications to CSEM. The synthetic aperture virtually increases the length of the aperture of a source by summing responses from multiple individual sources. Fan et al. (2010) initially demonstrate the increase in detectability from a steered synthetic aperture source array applied to CSEM responses. To create a beam to steer or focus, one weights the sources in the synthetic aperture source array; there are numerous algorithms from the radar field to determine the appropriate weights for a specific type of beam (Van Veen and Buckley, 1988). The synthetic aperture was only recently applied to CSEM fields because it was thought that diffusive fields could not be steered (Mandelis, 2000). Løseth et al. (2006) demonstrate that electromagnetic fields can be described by the wave and diffusion equations. A solution to the $3 \mathrm{D}$ scalar diffusion equation is a plane wave at single frequency with a defined direction of propagation (Løseth et al., 2006; Fan et al., 2010). The equation for synthetic aperture in the frequency domain at a single frequency is given by

$$
S(\mathbf{r})=\sum_{j} a_{j} F_{j}(\mathbf{r}),
$$

where $a_{j}$ is a complex weighting term and $F_{j}(\mathbf{r})$ is the response of any component of the electric or magnetic field for each source $j$ at the location $\mathbf{r}$.

For CSEM, implementing synthetic aperture is a postacquisition step and does not require any changes to the acquisition design. Others have approached extracting more subsurface information from CSEM responses by improving airwave removal, denoising, frequency differencing, inversion, and modeling (Amundsen et al., 2006; Nordskag and Amundsen, 2007; Abubakar et al., 2008; Maaø and Nguyen, 2010; Mattsson et al., 2012). One approach to enhance the reservoir response that manipulates CSEM responses similarly to synthetic aperture is focused-source electromagnetics (FSEM), in which four symmetric physical sources are linearly combined to simulate a vertical dipole (Davydycheva and Rykhlinski, 2009, 2011). The FSEM method uses focusing to build an ideal transmitter, which subdues irrelevant signals allowing more reservoir signal to reach the receiver (Davydycheva and Rykhlinski, 2011). Our method of the optimally weighted synthetic aperture also uses existing source locations to simulate a new source, but our method uses adaptive weights that focus or steer based on the response from the reservoir instead of forming a specific radiation pattern for the source. Because the data are acquired for each source separately, and the responses from each individual source are superposed numerically, we speak of a synthetic aperture source.

Fan et al. $(2011,2012)$ demonstrate steering and focusing with CSEM fields with a single towline. Generalizing to use sources from several towlines, one can choose the weights to steer the source array in the inline direction (along the towline), the crossline direction (perpendicular to the towline), or in both directions. Previously, we used exponential weighting in which we chose a single value for the amplitude term and a steering angle for the phase shift (Fan et al., 2011, 2012; Knaak et al., 2013). This type of weighting is analogous to a fixed beamformer for radar in which the weighting is independent of the signal (Van Veen and Buckley, 1988). The phase shift and amplitude terms for exponential weighting are linear in the spatial coordinates, which essentially forces the source array to radiate a plane wave. This type of weighting is not ideal for every situation. For example, a 2D source array centered over a reservoir would be more effective with weights that focus the energy toward the center. To achieve a less restrictive formulation, we define the weight as a complex number for each source. The new weighting creates an adaptive, weighted synthetic aperture source array in which the weight is allowed to take on any value. With this formulation, the number of weights corresponds to the number of sources in the synthetic aperture array. Previously, we tested different combinations of phase shifts and amplitude terms to find the best steering parameters with the range of steering angles and amplitudes set by what seemed reasonable based on the geometry. Testing the combinations is impractical given the large number of weights in the new formulation. Also, with a 2D source array, the functional form of the weights is not easily known. Focusing may be optimal for some source locations, although steering works better for others. To determine the optimal weights for a 2D source array, a new solving method is needed. In the next section, we introduce an optimization method used to find the optimal weights for the synthetic aperture source array.

\section{OPTIMIZING THE WEIGHTS FOR A SYNTHETIC APERTURE}

To ensure that a weighted synthetic aperture highlights the reservoir optimally for every source array location, we use optimization to solve for the weights used to steer the synthetic aperture source. The goal of applying a synthetic aperture to CSEM data is to increase the detectability of the reservoir and/or increase the spatial resolution. We measure the detectability as the magnitude of the difference between the pay field and the wet field. The pay field is the electromagnetic field recorded from a CSEM survey or the fields generated from a model including a reservoir. The wet field is the background field without the reservoir, also referred to as the dry field. To implement this method with real data, one needs an estimate of the response without the reservoir or the response from a nearby location without a reservoir. We apply weighted synthetic aperture to the pay and wet fields, as in equation 1, to determine the increase in the signal from the reservoir. The equations for the weighted synthetic aperture pay field and wet field are provided below:

$$
\begin{aligned}
& S^{p}(\mathbf{r})=\sum_{j} a_{j} F_{j}^{p}(\mathbf{r}), \\
& S^{w}(\mathbf{r})=\sum_{j} a_{j} F_{j}^{w}(\mathbf{r}),
\end{aligned}
$$

where $F_{j}^{p}(\mathbf{r})$ and $F_{j}^{w}(\mathbf{r})$ are any component of the electric or magnetic field at receiver $\mathbf{r}$ from the pay field and wet field, respectively, 
and $a_{j}$ is the weight for the source $j$. The difference gives the contribution from the secondary field created by the presence of the reservoir. This is the measure we use in the optimization scheme to determine the optimal weights. We do not need to know the location of the reservoir because the location of secondary field contains the necessary information needed to enhance the synthetic aperture response. The difference between the weighted synthetic aperture pay and wet responses is given by

$$
\Delta S(\mathbf{r})=S^{p}(\mathbf{r})-S^{w}(\mathbf{r}) .
$$

The optimal weights are those that maximize the difference between the two steered synthetic aperture responses. To ensure synthetic aperture amplifies the reservoir signal not noise, we suggest to use noiseless, modeled wet and pay responses, $S^{w}(\mathbf{r})$ and $S^{p}(\mathbf{r})$ in equation 4 , to calculate the optimal weights.

Another way to create a large, arbitrary difference between the responses is to use a set of weights equal to a large scalar value, which amplifies the magnitude of the response from each source in the synthetic aperture array. This type of weighting effectively increases the amount of energy radiating by the source array instead of increasing the signal from the reservoir. To ensure the energy radiated by the source array is fixed, we place the following constraint on the weights $a_{j}$ :

$$
\sum_{j}\left|a_{j}\right|^{2}=1
$$

These weights normalize the energy that is radiated by the synthetic aperture source. The following constrained optimization problem maximizes the difference between the pay and wet fields, while constraining the total energy radiated:

$$
\max |\Delta S(\mathbf{r})|^{2} \text { subject to } \sum_{j}\left|a_{j}\right|^{2}=1 .
$$

We define the optimal weights as those that create the maximum difference $\Delta S$ in the weighted synthetic aperture pay and wet responses at receiver location $\mathbf{r}$. The optimization gives higher amplitude to the sources with more information about the reservoir. We select the quadratic objective function in equation 6 , rather than the ratio between the pay and wet fields because it gives a linear system of equations for the weight $a_{j}$.

The optimization method we outline earlier is similar to the linear constrained optimization beamformers for synthetic aperture radar (Van Veen and Buckley, 1988). Both applications of beamforming include objective functions with measures of the signal of interest and linear constraints on the weights. For radar, the weights suppress any signal not from the defined direction of significance (Van Veen and Buckley, 1988). For CSEM, the weights amplify the portion of signal that contains from the reservoirs. The common way to solve this type of constraint optimization problem is to use Lagrangian multipliers, which could be used for our problem (Boas, 1983; Aster et al., 2005). However, because of the linearity of the objective function, we apply a different solving method. The quadratic term $|\Delta S(\mathbf{r})|^{2}$ in equation 6 is the equation for an ellipsoid in which the constraint $\sum_{j}\left|a_{j}\right|^{2}$ describes as a sphere. The optimal weights occur at the intersection of the sphere and the ellipsoid, which is the largest principal axis of the ellipsoid. Figure 1 depicts the geometry with 2D shapes. We can rewrite the inversion problem in quadratic form as

$$
\max \mathbf{a}^{\top} \mathbf{H a}^{*},
$$

where $*$ denotes the complex conjugate, $\mathbf{a}$ is the vector of optimal weights, and $\mathbf{H}$ is a Hermitian matrix, where the eigenvectors $\mathbf{u}$ of $\mathbf{H}$ are the columns of the matrix $\mathbf{U}$. The components of $\mathbf{H}$ are $\Delta F_{j} \Delta F_{k}^{*}$, the difference between the unweighted pay and wet fields, for $j=1, \ldots, n$ and $k=1, \ldots, n$ with $n$ equal to the number of sources in the synthetic aperture source array. The matrix is diagonalized to rotate to the principal axes of the ellipsoid by decomposing the Hermitian matrix into $\mathbf{H}=\mathbf{U} \boldsymbol{\Lambda} \mathbf{U}^{\top}$. The eigenvector $\mathbf{u}_{j}$ corresponding to the largest eigenvalue $\lambda_{\max }$ is the vector of optimal weights $\mathbf{a}$. We meet the weighting constraint by normalizing the vector of weights. One characteristic of this method is that the vector of weights multiplied by a constant phase shift remains a valid solution. This does give a known phase shift to the steered source, but it does not affect the overall steering created by the change in phase between sources.

The difference between wet and pay fields is equivalent to the imprint of the reservoir on the response. The amplitude of this signal is several magnitudes larger at small source-receiver offsets than at larger source-receiver offsets. The inversion focuses on the locations with higher magnitude in the difference of the response because the goal is to maximize the difference. However, there is valuable information in the signal at larger offsets. To force the inversion to value all the differences between responses evenly, the responses are weighted by the inverse of the amplitude of the wet field $F_{j}^{w}(\mathbf{r})$ as shown below:

$$
W_{j}(\mathbf{r})=1 /\left|F_{j}^{w}(\mathbf{r})\right| .
$$

We apply the weighting to each response from a source in the synthetic aperture array. This type of weighting is commonly used in inversion of CSEM data to equalize the amplitudes (Weitemeyer et al., 2010). The difference with evenly valued data is given by

$$
\Delta S_{j}(\mathbf{r})=W_{j}(\mathbf{r})\left(S_{j}^{p}(\mathbf{r})-S_{j}^{w}(\mathbf{r})\right) .
$$

Now, the optimization scheme finds the optimal weights that highlight the reservoir for each individual source, even those at large offsets. The optimization method solves for data-dependent weights that create an adaptive beamformer to maximize the signal from the

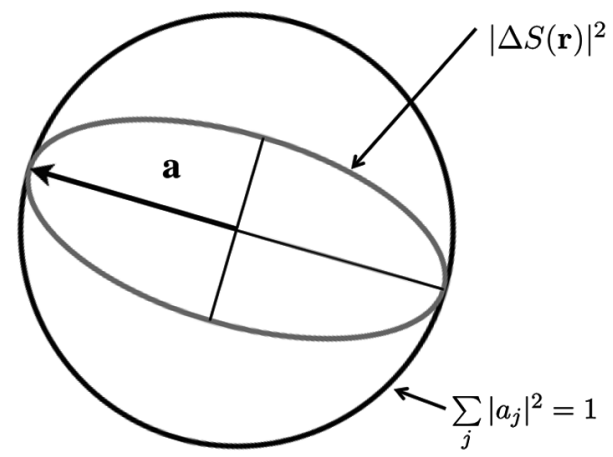

Figure 1. An illustration of the optimization problem depicted with 2D shapes. The squared absolute value of the difference is the equation for an ellipsoid, and the weighting constraint is the equation for a sphere. The vector that lies along the principal axis of the ellipsoid is the vector of optimal weights. 
reservoir encoded in the electromagnetic fields. The only inputs are a component of the electric or magnetic fields of the sources in the synthetic aperture array. The user decides on the length and width of the source array, which allows the method to work with any survey geometry. The optimization also independently switches from steering to focusing, depending on the geometry, without additional information from the user. For the application of this method to real data, one first needs an estimation of the background field. We suggest calculating the difference from wet and pay responses calculated from a synthetic model that approximates the subsurface at the survey location. To show the impact of these characteristics of the optimization method and the benefits of weighted synthetic aperture, we present two examples from modeled electromagnetic fields of two reservoirs in a shallow-water marine setting.

\section{SYNTHETIC EXAMPLES}

We present examples from a synthetic model to demonstrate the benefits of an optimized, steered synthetic aperture source array. The synthetic electromagnetic fields were generated with the IBCEM3D code, modeling software for 3D electromagnetic fields (Endo and Zhdanov, 2009). We modeled a shallow-water situation (water depth of $200 \mathrm{~m}$ ) with two reservoirs that are laterally separated. The model has an anisotropic layered background with typical vertical resistivities found in shallow-water locations, shown in Figure 2. The two reservoirs are $1.5-\mathrm{km}$ below the seafloor and $50 \mathrm{~m}$ thick with a resistivity of $50 \Omega \mathrm{m}$. The two reservoirs are separated $1.5 \mathrm{~km}$ laterally as shown in Figure 2. The survey design is a common industry CSEM survey setup with five towlines spaced $1.5 \mathrm{~km}$ apart, each with 186 source locations. The source is a 270-m horizontal electric dipole with a frequency of $0.2 \mathrm{~Hz}$. The 61 receivers are along one line, centered in the crossline direction, and spaced 500-m apart in the inline direction. A map view of the survey design is shown in Figure 3. To make our examples more realistic, we add a typical noise floor of $10^{-15} \mathrm{~V} / \mathrm{Am}^{2}$ independent, random, additive noise to the simulated electromagnetic fields (Constable, 2010). In this article, we only evaluate the suppression of additive noise, whereas the effect on multiplicative noise will be addressed in future work.

A benefit of the outlined weighted synthetic aperture technique is the flexibility of the method to work for several different applications. Here, we present two different applications of weighted synthetic aperture. The first example is for a situation in which a higher level of detectability is required. To increase the magnitude of the

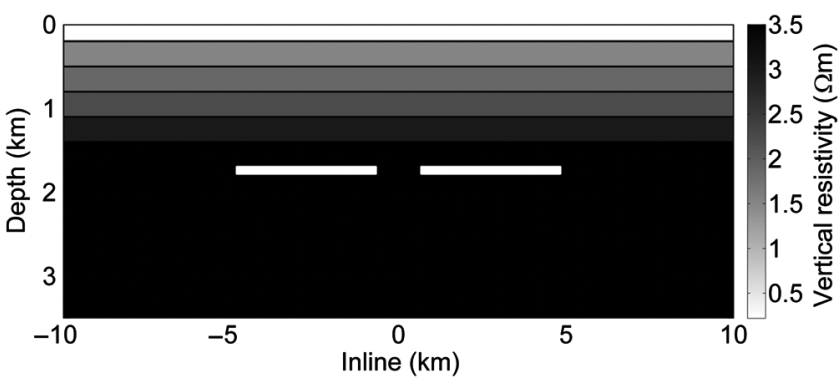

Figure 2. An inline cross section of the model used to generate the electromagnetic fields. The first layer is water with a depth of $200 \mathrm{~m}$. There are five sediment layers with varying resistivity. The reservoirs are shown as the white rectangles. The vertical scale is exaggerated. recorded anomaly, we apply a 2D weighted synthetic aperture source array. The second example is for a situation in which more information about the structure is needed. Resolving the two reservoirs in the model is best done with crossline steering only because the inline steering spatially averages the two anomalies. For these examples, we use only the inline component of the electric field. To view the electromagnetic fields, we use diagrams that display common midpoint, the lateral, spatial midpoint (ignoring depth differences) between the position of a receiver and a source, versus offset, the spatial distance between a receiver and a source. These diagrams show data points with common offsets along the horizontal lines. Displaying the response from the synthetic aperture array this way creates a pseudodepth section or an approximate vertical slice at the receivers through the inline-crossline plane as shown in Figure 3 (Silva Crepaldi et al., 2011). The difference is the measure of the response in the optimization method; however, it is more informative to view the normalized difference, which is the difference divided by the absolute value of the background field. Figure 4 shows the normalized difference of the modeled inline electric pay and wet fields with additive noise for no synthetic aperture (Figure 4a), 2D steered synthetic aperture (Figure 4b), and steered crossline only synthetic aperture (Figure 4c). For the synthetic inline electric response of the model described earlier, the anomaly from the reservoir appears at 7-km offset and the maximum of the anomaly is $27 \%$, which not only includes the signal from the reservoir but also additive noise. A typical criterion for detectability in CSEM surveys is a normalized difference of approximately $20 \%$ (Constable, 2010). With a 2D weighted synthetic aperture source array (shown in Figure 4b), the anomaly increases in magnitude and spatial area. However, the structural information about the two reservoirs is obscured. Applying synthetic aperture only in the crossline direction preserves the two anomalies (Figure 4c). The details of each example are described in the sections below.

\section{Increasing detectability}

If the goal of applying a synthetic aperture is to increase the signal from the reservoir, then the best method is to use a 2D synthetic aperture source because information from the crossline and inline directions is included. To apply the $2 \mathrm{D}$ synthetic aperture and find the optimal weights, we first decide on a length and width for the 2D synthetic aperture source array, formed from a spatial distribution of multiple sources. A larger synthetic aperture source array creates higher detectability, but the averaging in the inline direction also increases, which can obscure the structure. A smaller synthetic aperture source array preserves more information about the structure, but the application of beamforming is less effective. There is a trade-off between increasing detectability and maintaining a detect-

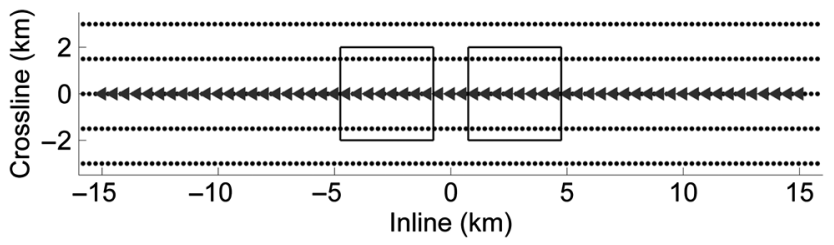

Figure 3. The survey geometry used to create the synthetic CSEM data. The sources are shown as black dots, and the receivers are shown as gray triangles. The locations of the reservoirs are outlined in black. 
able level of signal from the reservoir; a 2D synthetic aperture source formed from all source locations averages out the entire signal from the reservoir.

For this example, we arbitrarily use 21 sources in the inline direction and all 5 sources in the crossline direction. The resulting synthetic aperture source array is $5.7 \mathrm{~km}$ long and $6 \mathrm{~km}$ wide. The source spacing in the inline direction (270-m apart) is denser than the spacing in the crossline direction (1.5-km apart). Even with this discrepancy, we achieve coherent focusing in the crossline direction. We apply the optimization scheme to find the 105 weights that maximize the difference between the pay and wet fields for one source array location. We move the synthetic aperture source array around the entire survey footprint to simulate towing the 2D synthetic aperture source array. We make the assumption that, for a real data situation, the optimization scheme would use data generated from models of the expected structure, and hence the electromagnetic fields would not contain noise. Thus, inputs into the optimization method are the inline electrical response from each source included in the synthetic aperture array without noise. The optimization method finds the optimal weights for each source array location and for all receivers. We then apply the optimal weights to the noisy inline electrical pay and wet fields for each source in the $2 \mathrm{D}$ synthetic aperture array. The normalized difference of the inline electric fields for the 2D steered synthetic aperture source is shown in Figure 4b. With the application of the optimal weighted 2D synthetic aperture source, the anomaly from the reservoir has increased in magnitude and spatial extent when compared with the original

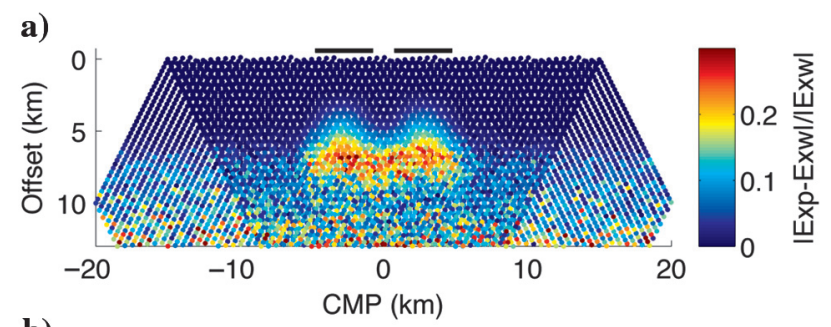

b)

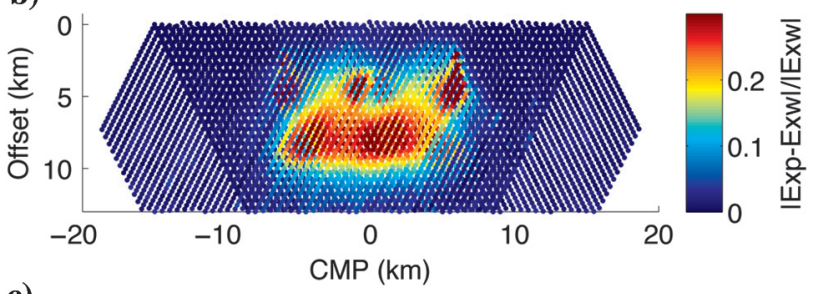

c)

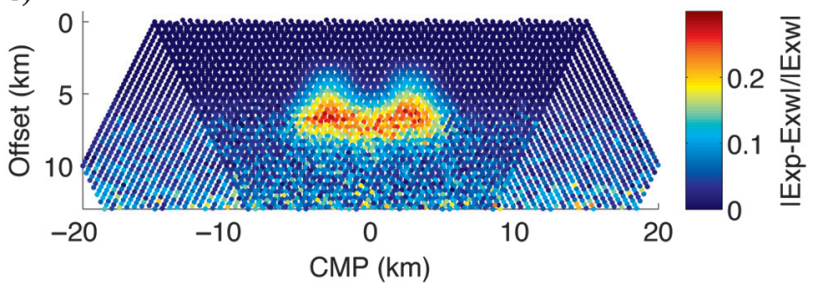

Figure 4. (a) The normalized difference of the inline electric pay field and the inline electric wet field with $10^{-15} \mathrm{~V} / \mathrm{Am}^{2}$ independent random noise added for the original data, (b) the optimal 2D steered synthetic aperture source array, and (c) the optimal crossline steered synthetic aperture source array. The two black bars above the top panel show the lateral extent of the two reservoirs as a function of the common midpoint. response. The maximum normalized difference is $46 \%$ (Figure 4b), which is an increase from the $27 \%$ anomaly in the original noisy data (Figure 4a). In addition, the additive noise, shown in the large offsets in the image without synthetic aperture, Figure 4a, does not appear in the normalized difference of the noisy inline electric fields from the steered 2D synthetic aperture source. There is still some noise in the image, but the addition of multiple sources in the synthetic aperture source array increases the magnitude of the coherent signal and almost completely stacks out the random additive noise.

To understand the adaptive nature of the optimization scheme, it is useful to look at the optimal weights for the $2 \mathrm{D}$ synthetic aperture source array from different locations. The weights correspond to the coefficients $a_{j}$ described in equation 7 . The complex, optimal weights for one source array location and receiver could be visualized by contour plots of the real and imaginary parts, or of the phase and amplitude. We choose the latter option because the phase and amplitude are better suited for a physical interpretation than are the real and imaginary parts. Figure $5 \mathrm{a}$ and $5 \mathrm{~b}$ displays contour plots of the amplitude and phase, respectively, of the steering coefficients for the source array centered at $-8.26 \mathrm{~km}$ and the receiver located in the center of the survey between the two reservoirs. The optimal weights for this location steer the field toward the center by giving a higher phase shift to the source farther away in the inline direction. In the crossline direction, the weights focus toward the center with a parabolic phase shift in which the outer towlines are weighted higher. The amplitude plot (Figure 5a) shows that the sources closer to the nearest reservoir, for this source array position, have a higher weight. The sources given lower amplitude weight contain fewer information about the reservoirs than those weighted higher. Figure $6 \mathrm{a}$ and $6 \mathrm{~b}$ displays contour plots of the amplitude and phase, respectively, for the source array centered at $3.08 \mathrm{~km}$. This source array location is directly over one reservoir, and the amplitude plot shows that more emphasis is placed on the source locations with larger offsets. Less emphasis on the sources over the reservoir is congruent with the expected weighting because small sourcereceiver offsets are dominated by the background signal (Constable, 2010). The phase shifts (Figure 6b) are similar to those from the other source array location but with a larger phase shift across the source array in the inline direction. Figures 5a and 6a show there is a section of the synthetic aperture array that has amplitude weighting close to zero, which demonstrates that the sources in that

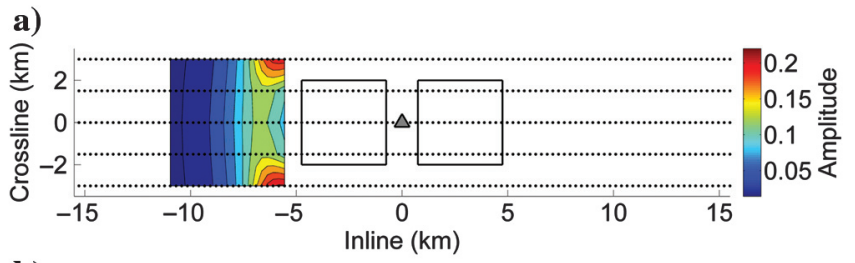

b)

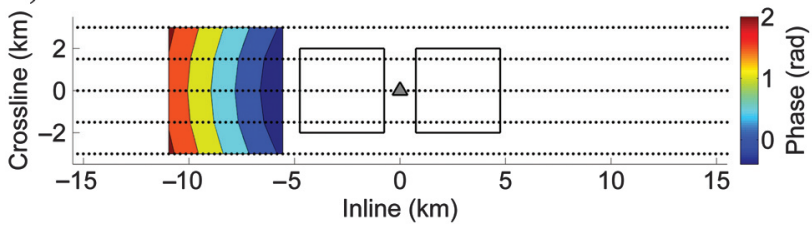

Figure 5. A map view of the (a) amplitude and the (b) phase of the optimal weights for the $2 \mathrm{D}$ synthetic aperture source array centered at $-8.26 \mathrm{~km}$ for the responses from the receiver specified by the gray triangle. 
part of the source array are not contributing to the increase in the anomaly from the reservoir. The optimization method essentially implemented a smaller synthetic aperture for these two source array locations, which indicates that we could have chosen a smaller synthetic aperture length. However, because the method is able to recognize when to reduce the length, there is no detriment from the longer synthetic aperture.

The optimal 2D weighted synthetic aperture source array increases the magnitude and spatial area of the anomaly. However, 2D steering averages the two anomalies into one large anomaly, which conceals the fact that there are two reservoirs present. The ability to discern if two reservoirs are present is difficult in the CSEM data. To increase the anomaly and retain the information about the structure, we apply a different steering method.

\section{Increasing lateral resolution}

It is often difficult with CSEM field responses to determine if a reservoir consists of one unit or two separate reservoirs. If the goal is to differentiate two bodies, then a crossline synthetic aperture is the best choice because the inline steering averages the two anomalies from the two receivers and the anomaly appears to be from one reservoir. We use sources from all five towlines to create a $6-\mathrm{km}-$ wide synthetic aperture source in the crossline direction. We apply the optimization method for the crossline synthetic aperture source array for all source array locations and receivers. The process is the same as for the 2D source array, but now we solve for five optimal weights for each source array instead of 105 . We apply the optimal weights to the inline electrical component of the pay and wet fields for each source in the crossline source array.

Figure $4 \mathrm{c}$ shows the normalized difference of the crosslineweighted inline electric fields. The two reservoirs are more discernible with crossline-weighted synthetic aperture than in the original data (Figure $4 \mathrm{a}$ ) or the normalized difference of the 2D steered inline electric fields (Figure 4b). The crossline-only synthetic aperture increases the magnitude and spatial localization of each individual reservoir and does not blur the two separate anomalies into one large anomaly. To quantify the improvement, we take the spatial average of the normalized difference from 6 to $8 \mathrm{~km}$ offset and 0.5 to $4 \mathrm{~km}$ common midpoint. The average normalized difference for the crossline weighted synthetic aperture field in this area is

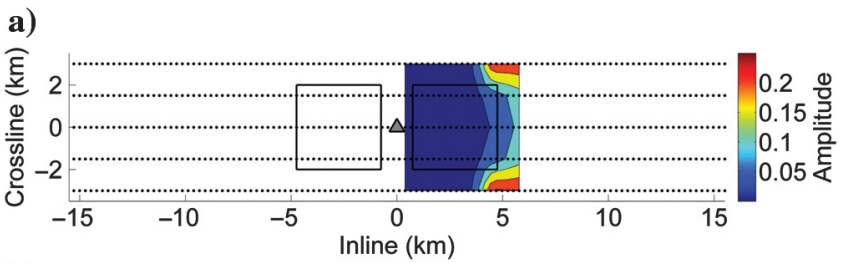

b)

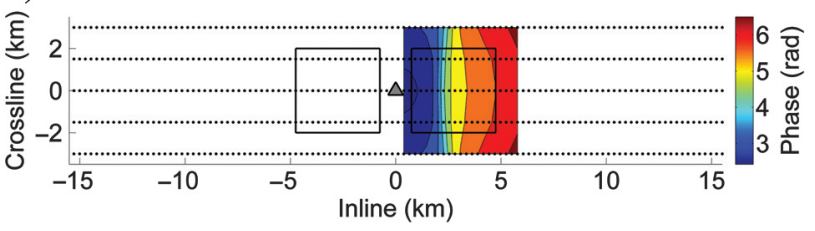

Figure 6. A map view of the (a) amplitude and the (b) phase of the optimal weights for the 2D synthetic aperture source array centered at $3.08 \mathrm{~km}$ for the response from the receiver specified by the gray triangle.
$21 \%$, whereas the same spatial average of the original field is $17 \%$. The additive noise is more visible in the crossline synthetic aperture fields (Figure 4c) than in the 2D steered fields (Figure 4b) because fewer sources are in the synthetic aperture source array, but the noise level is smaller than it is for the original data (Figure 4a). The optimal weights in the crossline direction create a focus by giving the sources farthest away larger phase shifts and amplitudes. Figure 7a shows the parabolic phase shifts for the optimal weights for the crossline source array located at $-17.44 \mathrm{~km}$, as the solid black line. Unlike Figures $5 \mathrm{~b}$ and $6 \mathrm{~b}$, we present these optimal weights in a crossline versus phase plot to view the parabolic shape, which cannot be seen with a contour plot. We did not require the optimization to create a focus, but the inversion found the best weights for the situation. We can verify if these weights are reasonable by analytically calculating the phase shifts for each of the five sources to focus the field on the reservoir. The equation for a phase shift to create a focus is given by (Fan et al., 2011)

$\Phi(x, y, z)=k\left(\sqrt{\left(x-x_{f}\right)^{2}+\left(y-y_{f}\right)^{2}+\left(z-z_{f}\right)^{2}}-D\right)$,

where $\left(x_{f}, y_{f}, z_{f}\right)$ is the location of focus; $k$ is the wavenumber; and $D$ is the distance from the focal point to the nearest end of the synthetic aperture, which normalizes the phase shift. To use equation 10, we assume a homogeneous field and a single resistivity. We calculate the wavenumber for the survey frequency, $0.2 \mathrm{~Hz}$, and a resistivity equal to $3 \Omega \mathrm{m}$ (the resistivity of the second-to-last layer in our model), and we set the depth of the focus at $1.51 \mathrm{~km}$, which is the depth of the reservoirs. The focus point varies for each source array location. We choose one source array location $(-17.44 \mathrm{~km})$ to compare the optimal focusing with the calculated focusing.

The calculated focus point that produces a curvature matching the optimal weights is $\left(x_{f}, y_{f}, z_{f}\right)=(-2.21 \mathrm{~km}, 0 \mathrm{~km}, 1.51 \mathrm{~km})$. The phases of the optimal weights and the calculated weights are shown

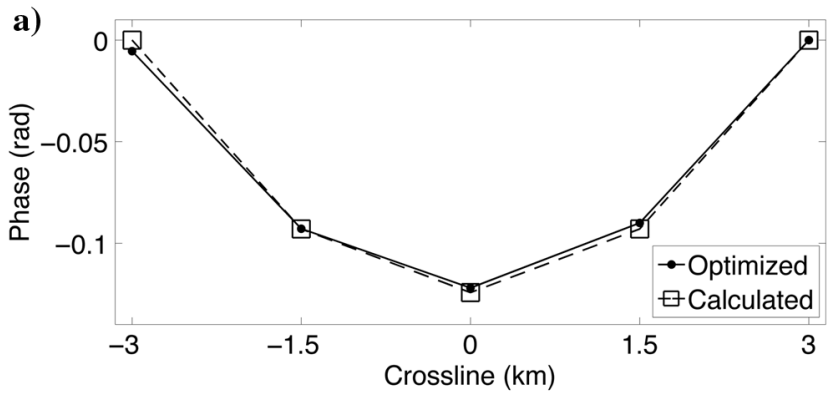

b)

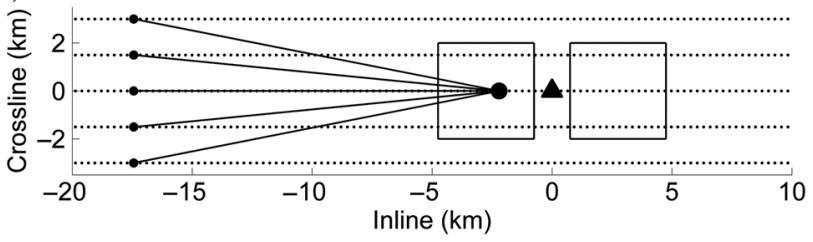

Figure 7. (a) The phase of the optimal and calculated weights for a crossline synthetic aperture source array located at $-17.44 \mathrm{~km}$. (b) The calculated focus point (the circle over the left reservoir) for the crossline synthetic aperture created from the five sources shown as black dots for a receiver located in the center of the survey. 
in Figure 7a, and the location of the sources and the calculated focus point are shown in Figure $7 \mathrm{~b}$. The focus point is only an estimate of where the optimal weights focus point is located because we assume in the calculation a homogeneous resistivity model for the calculation. We find the phase for the calculated focus point that almost identically matches the curvature of the phase of the optimal weights and the spatial location of calculated focus point is reasonable for the geometry of the survey, which demonstrates that the optimal weights agree with the analytical focusing. Other source array locations have similar reasonable analytical focus point locations between or over the reservoirs. The optimization method thus solves for the weights that correspond to the optimal focus point for each source array location without any additional inputs from the user. In this example, there are five towlines symmetric about the reservoirs, which make focusing the best weighting option. The steering or focusing created by the optimal weights depends on the geometry of the survey, the information within the responses, and the size of the array.

The success of optimally weighted synthetic aperture applied to CSEM depends on the amount of information about the reservoir encoded in the measurements. In addition, the accuracy of the weights will depend on the accuracy of the modeled response by the user. In recent work, Knaak (2015) presents results from tests of this optimization method on inaccurate models, which show that increases in detectability occur even with incorrect models.

\section{CONCLUSION}

Locating smaller, deeper, or shallow-water targets with CSEM in complicated environments is becoming more challenging. We have demonstrated the benefits of applying the technique of synthetic aperture, which virtually increases the length, and/or width of the source. Applying weights to the synthetic aperture source array allows us to steer or focus the array in the inline and crossline directions. With complex settings and more intricate survey geometries, the best type of weighting is no longer intuitive. In a less complicated environment, one could possibly define a single steering angle or focusing point for every source array location, but this is not the optimal weighting for all survey designs and environments. We presented a method to optimize the weights for synthetic aperture source arrays, which acts as an adaptive beamformer by adjusting to the information about the reservoir encoded in the CSEM data. A 2D synthetic aperture source array applied to CSEM data increases the detectability of the reservoir and reduces additive noise but may obscure structure. We found that applying crossline weighting to noisy inline electric fields from a model with two laterally separated reservoirs preserved the structure, increased the magnitude of the anomalies from the reservoirs, and reduced the noise.

We apply a synthetic aperture to increase the anomaly from a reservoir in imaging. The best way to recover a model of the subsurface from CSEM data is through inversion. An inversion of the weighted $2 \mathrm{D}$ synthetic aperture source response may produce a more accurate model of the subsurface than the original data. However, the impact of the weighted synthetic aperture on inversion results is unknown. Future work will explore if applying a weighted synthetic aperture before inversion increases the accuracy of the recovered model. We will also continue to work with the synthetic aperture for the forward problem by testing the technique on more complicated models. Other future work includes testing the benefit of synthetic aperture on CSEM responses with multiplicative noise and possibly impacts of synthetic aperture to the CSEM survey design.

\section{ACKNOWLEDGMENTS}

We thank E. Gasperikova, S. Davydycheva, and T. Wiik for their critical and constructive comments. We acknowledge the use of synthetic data generated from IBCEM3D, a modeling code developed by the CEMI Consortium at the University of Utah. Finally, we are grateful for the financial support from the Shell GameChanger Program of Shell Research, and we thank Shell Research for the permission to publish this work.

\section{REFERENCES}

Abubakar, A., T. M. Habashy, V. L. Druskin, L. Knizhnerman, and D. Alumbaugh, 2008, 2.5D forward and inverse modeling for interpreting low frequency electromagnetic measurements: Geophysics, 73, no. 4, F165F177, doi: 10.1190/1.2937466.

Amundsen, L., L. Lseth, R. Mittet, S. Ellingsrud, and B. Ursin, 2006, Decomposition of electromagnetic fields into upgoing and downgoing components: Geophysics, 71, no. 5, G211-G223, doi: 10.1190/1.2245468.

Aster, R. C., C. H. Thurber, and B. Borchers, 2005, Parameter estimation and inverse problems: Elsevier Academic Press, International Geophysics Series 90.

Boas, M., 1983, Mathematical methods in the physical sciences 2nd ed.: Wiley.

Constable, S., 2010, Ten years of marine CSEM for hydrocarbon exploration: Geophysics, 75, no. 5, 75A67-75A81, doi: 10.1190/1.3483451.

Constable, S., and L. J. Srnka, 2007, An introduction to marine controlledsource electromagnetic methods for hydrocarbon exploration: Geophysics, 72, no. 2, WA3-WA12, doi: 10.1190/1.2432483.

Davydycheva, S., and N. Rykhlinski, 2009, Focused-source EM survey versus time-domain and frequency-domain CSEM: The Leading Edge, 28, 944-949, doi: 10.1190/1.3192841.

Davydycheva, S., and N. Rykhlinski, 2011, Focused-source electromagnetic survey versus standard CSEM: 3D modeling in complex geometries: Geophysics, 76, no. 1, F27-F41, doi: 10.1190/1.3511353.

Edwards, N., 2005, Marine controlled source electromagnetics: Principles, methodologies, future commercial applications: Surveys in Geophysics, 26, 675-700, doi: 10.1007/s10712-005-1830-3.

Eidesmo, T., S. Ellingsrud, L. M. MacGregor, S. Constable, M. C. Sinha, S. Johansen, F. N. Kong, and H. Westerdahl, 2002, Sea bed logging (SBL), a new method for remote and direct identification of hydrocarbon filled layers in deepwater areas: First Break, 20, 144-152, doi: 10.1046/j $.1365-2397.2002 .00264 . x$

Endo, M., and M. Zhdanov, 2009, IBCEM3D, http://www.cemi.utah.edu/ soft/index.html, accessed 12 July 2011.

Fan, Y., R. Snieder, E. Slob, J. Hunziker, and J. Singer, 2011, Steering and focusing diffusive fields using synthetic aperture: EPL (Europhysics Letters), 95, 34006.

Fan, Y., R. Snieder, E. Slob, J. Hunziker, J. Singer, J. Sheiman, and M. Rosenquist, 2010, Synthetic aperture controlled source electromagnetics: Geophysical Research Letters, 37, L13305, doi: 10.1029/2010GL043981.

Fan, Y., R. Snieder, E. Slob, J. Hunziker, J. Singer, J. Sheiman, and M. Rosenquist, 2012, Increasing the sensitivity of controlled-source electromagnetics with synthetic aperture: Geophysics, 77, no. 2, E135-E145, doi: 10.1190/geo2011-0102.1.

Jensen, J., S. I. Nikolov, K. L. Gammelmark, and M. H. Pedersen, 2006, Synthetic aperture ultrasound imaging: Ultrasonics, 44, e5-e15, doi: 10.1016/j.ultras.2006.07.017.

Knaak, A., 2015, 3D synthetic aperture for controlled-source electromagnetics: Ph.D. thesis, Colorado School of Mines.

Knaak, A., R. Snieder, Y. Fan, and D. Ramirez-Mejia, 2013, 3D synthetic aperture and steering for controlled-source electromagnetics: The Leading Edge, 32, 972-978, doi: 10.1190/tle32080972.1.

Løseth, L. O., H. M. Pedersen, B. Ursin, L. Amundsen, and S. Ellingsrud, 2006, Low-frequency electromagnetic fields in applied geophysics: Waves or diffusion?: Geophysics, 71, no. 4, W29-W40, doi: 10.1190/ 1.2208275 .

Maaø, F., and A. Nguyen, 2010, Enhanced subsurface response for marine CSEM surveying: Geophysics, 75, no. 3, A7-A10, doi: 10.1190/1 .3377054 .

Mandelis, A., 2000, Diffusion waves and their uses: Physics Today, 53, 29-34, doi: 10.1063/1.1310118. 
Mattsson, J., P. Lindqvist, R. Juhasz, and E. Bjrnemo, 2012, Noise reduction and error analysis for a towed EM system: 82nd Annual International Meeting, SEG, Expanded Abstracts, 795-799.

Nordskag, J., and L. Amundsen, 2007, Asymptotic airwave modeling for marine controlled-source electromagnetic surveying: Geophysics, 72, no. 6, F249-F255, doi: 10.1190/1.2786025.

Silva Crepaldi, J., M. Pereira Buonora, and I. Figueiredo, 2011, Fast marine CSEM inversion in the CMP domain using analytical derivatives: Geophysics, 76, no. 5, F303-F313, doi: 10.1190/geo2010-0237.1.
Van Veen, B., and K. Buckley, 1988, Beamforming: A versatile approach to spatial filtering: IEEE ASSP Magazine, 5, no. 2, 4-24, doi: 10.1109/53 .665 .

Weitemeyer, K., G. Gao, S. Constable, and D. Alumbaugh, 2010, The practical application of 2D inversion to marine controlled-source electromagnetic data: Geophysics, 75, no. 6, F199-F211, doi: 10.1190/1.3506004 